\title{
Summary of the Origin and Development of Fragrant Medicine and Incense Culture
}

\author{
Haiyan Wang, Yajun Li* \\ Shaanxi University of Traditional Chinese Medicine, Xianyang 712046, Shaanxi Province, China \\ *Corresponding author: Yajun Li, liyajun@vip.126.com
}

\begin{abstract}
Fragrant medicine is a special kind of Chinese medicine, with complex sources and multiple functions. Fragrant medicine has captured the incense culture in this application, and this culture has spread overseas, as well as giving a huge impact. This article bases the history of transparent medicine and incense culture in an attempt to conquer the value of developing fragrant medicine and incense while seeking cultural roots.
\end{abstract}

Keywords: Fragrant medicine; Incense culture

Publication date: July 2021; Online publication: July 31, 2021

\section{Introduction}

The Chinese fragrant medicine culture is derived from the traditional Chinese medicine (TCM) culture. It is unique, diverse in form and rich in connotation. It is sublimated from a phenomenon to a concept, and as a special cultural carrier, it carries the philosophy of respecting heaven and pleasing people. It is of great significance to better explore and develop fragrant medicine, to achieve the development and inheritance of fragrant medicine and fragrant culture, also to understand the origin and development of fragrant medicine.

\section{Fragrant medicine and the origin of fragrance traceability}

Fragrant Medicine originated very early, "the ancestors were exorcised from pests, and haze appeared repeatedly." The ancestors chose water to live, but insects and rodents bred more and infected people, the mildly infected ones got sick, the more severe ones developed into infectious diseases, affecting the survival of the entire ethnic group. "Feng Su Tong" states that, "the ancestors lived in grass and were often poisoned by this poison, so Xiang Wen Lao said that he was safe. "Modern science of infectious disease clearly has a variety of infectious diseases infected and transmitted by mosquitoes, such as dengue fever, malaria, yellow fever, Japanese encephalitis, etc." Ancestors discovered that the smell of burning plants can repel mosquitoes and prevent diseases, thus they consciously burned aromatic plants with special smells. "Zhou Li" contains "smearing from the wild grass, all the things of the worms;" "The Book of Songs" describes "smearing rats in the dome and stuffing them to Jinhu." Gu Xian observed the details and concluded that fragrant medicine can also prevent the plague. "Natural History" in 99 BC, "Chang'an epidemic, burns the fragrance of the soul, the sick in the palace will rise immediately after the smell, and those who are less than three days old will suffer from the disease." This method has been used in plague prevention and to control in successive dynasties.

Throughout the history of Chinese plague prevention and control, fragrant medicine is indispensable. Modern studies have proved that aromatic medicines are rich in volatile oils, also have the effect of inhibiting and killing germs and viruses. In the prevention and control of the new crown epidemic, 
aromatic medicine has also contributed its special strength.

\section{The purpose, use form and modern research of fragrant medicine}

Fragrances are widely used to repel mosquitoes, mice and ants, purify the air, also prevent filth and epidemics. Through practice, medical experts in the past have continuously summarized and discovered the types and uses of fragrances. People have increasingly more fragrances, from Chinese origin than foreign countries. Imported, since the beginning of the "Ben Jing," there have been records of herbal and medical works in all dynasties. The fragrant medicine smells pleasant, and the spicy fragrance spreads. It has many functions such as strengthening the spleen and liver, calming the heart and nerves, removing dampness, refreshing the brain and resuscitation, regulating Qi and promoting blood circulation, dredging collaterals and relieving pain.

It can be smoked and ignited, also be worn with you, the "Shan Hai Jing" contains, the name is smoked grass, "as smelly as Wuwu, wearing it can prevent furunculosis, you can put it in the incense box or a sachet, hang in the house," "Book of Rites" states that, "Men and women, both admire the smell of incense," Chen Jing's note states that, "The smell of incense, the fragrance is also, the sachet of later generations is its legacy." In recent years, fragrant medicine has been used in the treatment of depression, and the effect is remarkable.

\section{Origin of incense culture}

The incense can be traced back to the late Neolithic period. Burn wood and raise smoke, smell it in air, and sacrifice to heaven and earth. The firewood in the burning festival is the most primitive image of incense. Sacrifice is one of the most important rituals in a country, nation, and family. Through the sacrificial ritual, incense becomes a symbol of ritual. National sacrifices, family sacrifices, religions, different occasions and identities, all use different incense and regulations.

Yi Zhang ${ }^{[1]}$ summed up the history of traditional Chinese incense culture as "beginning in the spring and autumn, growing in the Qin and Han Dynasties, complete in the Sui and Tang Dynasties, and prosperous in the Song Dynasty." With the development and progress of human society, the pursuit of material richness and spiritual level has given fragrant medicine a more beautiful meaning.

\section{Important historical figures in fragrant medicine and incense culture}

In the history of the development of fragrant medicine culture, "Yuan Qu, Ye Fan, Wei Ding, Tingjian Huang" are not to be forgotten. The spirit and soul of fragrant medicine comes from Yuan Qu. The first monograph on fragrant medicine comes from Ye Fan. The "Tianxiang Biography" written by Wei Ding is the first special document of agarwood; Tingjian Huang summarized the cultural connotation of incense, "the ten virtues of incense." It has a profound impact on the formation and development of incense roads in countries all over the world.

Yuan Qu loves fragrant medicine; this is reflected in the types and quantity of fragrant medicines in his works. Qixia Li ${ }^{[2]}$ summarized the vanilla fragrant woods in "The Songs of the Chu" total 34 kinds, including 22 kinds of vanilla and 12 kinds of fragrant wood, which were never been discovered before "The Songs of Chu." The Kingdom of Chu worshipped the gods and spirits, and Yuan Qu would inevitably be affected in his creation. For example, Hui Zhao ${ }^{[3]}$ believed that in Yuan Qu "there is a general psychology of pursuing longevity in the deep consciousness," and vanilla is more of "used by the gods' family to lift the flying fairy immortal medicine ${ }^{[3]}$."

Ye Fan was talented, extensively involved in literature, and history. In addition to Ye Fan, the Southern Dynasty historian, Yue Shen wrote a volume of "Hexiang Fang" and "Mixiang Gaofang." The original book was lost, only the preface of "Hexiang Fang" was recorded in "Song Shu, Ye Fan 
Biography." "Musk 
is too taboo, too much will do harm. It's easy to be calm." Ye Fan used incense as a metaphor for people, and was very familiar with the nature and taste of fragrance, also the use of taboos. Unlike Yuan Qu, Ye Fan sighed with the lack of fragrance. Bei Luo ${ }^{[4]}$ and others believed that "Hexiang Fang" is China's first monograph on fragrant medicine.

The Song Dynasty was the heyday of the development of Chinese incense culture, Wei Ding said that he was relegated to Hainan in his later years and wrote "Tianxiang Biography." "Tianxiang Biography" is the first document to evaluate and appraise the quality of agarwood. It discusses the origin of agarwood and the distinction between its advantages and disadvantages. It classifies and ranks Hainan agarwood from the ways of smell, appearance, and generation, and summarizes "four names and 20 shapes," putting forward the evaluation criteria of "clear, profound and long-lasting" agarwood smell, which laid the foundation for the research and evaluation standards of agarwood for later generations ${ }^{[4]}$.

\section{Conclusion}

Fragrant medicine originated from TCM, closely related to the Chinese society. Firstly, it is medicine to repel insects and epidemics, as well as to protect the body. Secondly, leisurely and elegantly in the use of meditation, to remove irritability and trouble. It can be said that the Chinese incense culture takes incense as its companion, ritual as its bone, and virtue as its soul.

\section{Disclosure statement}

The author declares no conflict of interest.

\section{References}

[1] Zhang Y, 2006, Looking at the Revival of Chinese Traditional Incense Culture from Japan's "Xiangdao." Gansu Agriculture, 12:281.

[2] Li Q, 2016, The Chongxiang and Pre-Qin Fragrant Culture in "Chu Ci." Journal of Changchun University of Science and Technology (Social Science Edition), 29(05): 105-108+129.

[3] Zhao H, 1989, Immortals, Medicine, Chu Ci. Zhongzhou Academic Journal, (03): 96-100.

[4] Luo B, Yang Y, He R, et al., 2019, The Formation and Development of Agarwood Culture. Journal of Southwest Forestry University (Social Science), 3(04): 43-46. 